\title{
Preface to the Special Issue on "Heterogeneous Photocatalysts: From Fundamentals to Innovative Applications"
}

\author{
Dai-Viet N. Vo ${ }^{1}$ Van-Huy Nguyen ${ }^{2} \cdot$ Leonardo Palmisano $^{3} \cdot$ Chao-Wei Huang ${ }^{4} \cdot$ Sonil Nanda ${ }^{5} \cdot$ Trinh Duy Nguyen $^{1}$
}

Published online: 28 October 2020

(c) Springer Science+Business Media, LLC, part of Springer Nature 2020

Heterogeneous photocatalysis, the subject of this Special Issue, is a versatile, low-cost and environmentally benign technology. Hence, there are significant progress and efforts to exploring heterogeneous photocatalysts, which opens new avenues for a wide range of industrial photocatalytic applications. Herein, this thematic issue of Topics in Catalysis is a collection of 36 manuscripts from globally recognized scholars and researchers with the focus on emerging trends in heterogeneous photocatalysis. Apart from the fundamentals in photocatalysis, i.e., mechanism and kinetics, this Special Issue also emphasizes the industrial and agricultural wastewater remediation, air pollutant treatment, as well as chemicals and green fuel production, viz., hydrogen via various photocatalytic processes from lab-scale to pilot-scale studies. Moreover, advanced material preparation approaches, namely, microwave-assisted solvothermal synthesis, modified sol-gel method, and supercritical anti-solvent precipitation route for synthesizing nano-photocatalysis, wastederived nanomaterials, and nanocomposites, are highlighted. In addition to examining operational parameters, photocatalytic process optimization, modeling and advanced photoreactor coupled with other biological processes are underlined in this notable collection. We believe that this thematic issue

Dai-Viet N. Vo

vndviet@ntt.edu.vn; vo.nguyen.dai.viet@gmail.com

Van-Huy Nguyen

vhnguyen@lhu.edu.vn

Leonardo Palmisano

leonardo.palmisano@unipa.it

Chao-Wei Huang

huangcw@nkust.edu.tw

Sonil Nanda

sonil.nanda@usask.ca

Trinh Duy Nguyen

ndtrinh@ntt.edu.vn

1 Center of Excellence for Green Energy and Environmental Nanomaterials (CE@GrEEN), Nguyen Tat Thanh of Topics in Catalysis on "Heterogeneous Photocatalysts: From Fundamentals to Innovative Applications" can successfully provide an informative forum for scientists, engineers, physicists, and chemists to rapidly address the most critical and emerging issues in the field of heterogeneous photocatalysis.

Finally, the Guest Editors would like to appreciate all the contributors worldwide due to their impactful and outstanding publication in this Special Issue. The dedicated time and constructive comments from the reviewers to appraise and improve the quality of submitted manuscripts are also indebted. Additionally, we are grateful to the Editor-in-Chief of Topics in Catalysis, Prof. Gabor A. Somorjai and Editorial Board Member Prof. Detlef W. Bahnemann for their noteworthy guidance towards the successful completion of this topical collection. The untiring and persistent assistance from Mr. Matthew Smyllie and Ms. Gursimaran Kaur of the Journal Editorial Office during the accomplishment of this thematic issue is greatly appreciated.

Publisher's Note Springer Nature remains neutral with regard to jurisdictional claims in published maps and institutional affiliations.

University, 300A Nguyen Tat Thanh, District 4, Ho Chi Minh City 755414, Vietnam

2 Key Laboratory of Advanced Materials for Energy and Environmental Applications, Lac Hong University, Bien Hoa 810000, Vietnam

3 Dipartimento di Ingegneria, Università di Palermo, Viale delle Scienze, 90128 Palermo, Italy

4 Department of Chemical and Materials Engineering, National Kaohsiung University of Science and Technology, Kaohsiung 80778, Taiwan

5 Department of Chemical and Biological Engineering, University of Saskatchewan, Saskatoon, Saskatchewan S7N 5A9, Canada 